\title{
A two-Higgs-doublet model facing experimental hints
}

\author{
Andreas Crivellin ${ }^{1, a}$, Julian Heeck ${ }^{2, b}$, and Peter Stoffer ${ }^{3, c}$ \\ ${ }^{1}$ CERN Theory Division, $\mathrm{CH}-1211$ Geneva 23, Switzerland \\ ${ }^{2}$ Service de Physique Théorique, Université Libre de Bruxelles, Boulevard du Triomphe, CP225, 1050 Brussels, Belgium \\ ${ }^{3}$ Helmholtz-Institut für Strahlen- und Kernphysik (Theory) and Bethe Center for Theoretical Physics, University of Bonn, D-53115 \\ Bonn, Germany
}

\begin{abstract}
Physics beyond the Standard Model has so far eluded our experimental probes. Nevertheless, a number of interesting anomalies have accumulated that can be taken as hints towards new physics: BaBar, Belle, and $\mathrm{LHCb}$ have found deviations of approximately $3.8 \sigma$ in $B \rightarrow D \tau v$ and $B \rightarrow D^{*} \tau v$; the anomalous magnetic moment of the muon differs by about $3 \sigma$ from the theoretic prediction; the branching ratio for $\tau \rightarrow \mu \nu v$ is about $2 \sigma$ above the Standard Model expectation; and CMS and ATLAS found hints for a non-zero decay rate of $h \rightarrow \mu \tau$ at $2.6 \sigma$. Here we consider these processes within a lepton-specific two-Higgs doublet model with additional non-standard Yukawa couplings and show how (and which of) these excesses can be accommodated.
\end{abstract}

\section{Introduction}

This talk is based on Ref. [1], where a more detailed discussion can be found. Tests of flavor universality or flavor violation serve as a useful tool to search for physics beyond the Standard Model (SM), seeing as the SM predictions are precisely known. Some experiments have reported on deviations from the SM, which we list below.

- Lepton universality in semileptonic $B$ decays, parametrized by the ratio

$$
\mathcal{R}\left(D^{(*)}\right) \equiv \frac{\mathrm{BR}\left(B \rightarrow D^{(*)} \tau v\right)}{\mathrm{BR}\left(B \rightarrow D^{(*)} \ell v\right)}, \quad \ell=e, \mu,
$$

has been studied by BaBar, Belle and LHCb. Combining their experimental values yields

$$
\mathcal{R}(D)_{\exp }=0.388 \pm 0.047, \quad \mathcal{R}\left(D^{*}\right)_{\exp }=0.321 \pm 0.021 \text {. }
$$

Together, these values deviate by more than $3 \sigma$ from the SM prediction [2-4]

$\mathcal{R}(D)_{\mathrm{SM}}=0.297 \pm 0.017, \quad \mathcal{R}\left(D^{*}\right)_{\mathrm{SM}}=0.252 \pm 0.003$.

One possible new-physics explanation comes in the form of a charged scalar [5-7].

- The muon's anomalous magnetic moment $a_{\mu} \equiv(g-$ $2)_{\mu} / 2$, as measured by the Brookhaven experiment E821 [8], deviates by $2.7 \sigma$ from its SM value,

$$
a_{\mu}^{\exp }-a_{\mu}^{\mathrm{SM}}=(236 \pm 87) \times 10^{-11} .
$$

\footnotetext{
a e-mail: andreas.crivellin @ cern.ch

be-mail: julian.heeck@ulb.ac.be

$\mathrm{c}_{\mathrm{e}-\mathrm{mail}}$ stoffer@hiskp.uni-bonn.de
}

It is not yet clear if this discrepancy is due to new physics or rather underestimated hadronic uncertainties; there are ongoing efforts to reduce the model dependence in the hadronic light-by-light estimate based on dispersion relations [9-12] or lattice QCD [13-16]. A possible interpretation in terms of new physics has been found in two-Higgs-doublet models (2HDM) [17, 18], in particular the lepton-specific 2HDM [19-22].

- For $\tau \rightarrow \mu v v$, the dominant uncertainty in the SM prediction for the branching ratio comes from the $\tau$ lifetime. Using the PDG [23] values for $\tau$ lifetime and branching ratios

$$
\begin{aligned}
& B_{\mu} \equiv \mathrm{BR}(\tau \rightarrow \mu \bar{v} v)_{\exp }=(17.41 \pm 0.04) \%, \\
& B_{e} \equiv \mathrm{BR}(\tau \rightarrow e \bar{v} v)_{\exp }=(17.83 \pm 0.04) \%,
\end{aligned}
$$

we can determine the deviations from the SM prediction $\Delta_{\ell} \equiv B_{\ell} / B_{\ell}^{\mathrm{SM}}-1[24]$ as

$$
\Delta_{\mu}^{\mathrm{PDG}}=(0.69 \pm 0.29) \%, \quad \Delta_{e}=(0.28 \pm 0.28) \% .
$$

There is a deviation of about $2.4 \sigma$ for the muon final state, whereas the electron channel is compatible with the SM prediction, hinting at lepton non-universality. Charged scalars of a 2 HDM will modify the rate [2426].

- Going from lepton non-universality to outright lepton flavor violation, we are drawn to the recent CMS excess of $2.4 \sigma$ in $h \rightarrow \mu \tau$ [27]:

$$
\mathrm{BR}(h \rightarrow \mu \tau)=\left(0.84_{-0.37}^{+0.39}\right) \% .
$$

A similar search at ATLAS [28] finds $\operatorname{BR}(h \rightarrow \mu \tau)=$ $(0.77 \pm 0.62) \%$, which slightly bumps the significance to about $2.6 \sigma$. Possible explanations naturally require an extended scalar sector [18, 29-35]. 
Each anomaly individually can be accommodated in SM extensions by scalars, e.g. 2HDMs. The goal of our study is to see if all four anomalies can be explained simultaneously with a fairly minimal model.

\section{Modified 2HDM-X}

We will study a lepton-specific 2HDM (2HDM-X), defined by the Yukawa couplings in the Lagrangian

$$
\mathcal{L}_{Y}=-\bar{Q}_{L} Y^{u} \tilde{\Phi}_{2} u_{R}-\bar{Q}_{L} Y^{d} \Phi_{2} d_{R}-\bar{L}_{L} Y^{\ell} \Phi_{1} e_{R}+\text { h.c. },
$$

with additional couplings that break the type-X structure

$$
\Delta \mathcal{L}_{Y}=-\bar{Q}_{L} \xi^{u} \tilde{\Phi}_{1} u_{R}-\bar{Q}_{L} \xi^{d} \Phi_{1} d_{R}-\bar{L}_{L} \xi^{\ell} \Phi_{2} e_{R}+\text { h.c. }
$$

The scalar interactions with fermions after electroweak breaking can be written as

$$
\begin{aligned}
\mathcal{L} \supset & \bar{v}_{i} \Gamma_{v_{i} \ell_{j}}^{H^{+}} P_{R} \ell_{j} H^{+} \\
& +\bar{u}_{i}\left(\Gamma_{u_{i} d_{j}}^{H^{+}} R L P_{L}+\Gamma_{u_{i} d_{j}}^{H^{+} L R} P_{R}\right) d_{j} H^{+} \\
& +\sum_{H_{k}^{0}=h, A, H} \sum_{f=u, d, \ell}\left(\bar{f}_{i} \Gamma_{f_{i} f_{j}}^{H_{1}^{0} L R} P_{R} f_{j} H_{k}^{0}\right)+\text { h.c. }
\end{aligned}
$$

where the couplings - in the limit of large $\tan \beta$ of interest here - are given by

$$
\begin{aligned}
\Gamma_{q_{i} q_{j}}^{h L R} & \simeq-\frac{1}{\sqrt{2}}\left(\frac{m_{q_{i}}}{v} \delta_{i j} \cos \alpha-\epsilon_{i j}^{q} \sin \alpha\right), \\
\Gamma_{q_{i} q_{j}}^{H L R} & \simeq-\frac{1}{\sqrt{2}}\left(\frac{m_{q_{i}}}{v} \delta_{i j} \sin \alpha+\epsilon_{i j}^{q} \cos \alpha\right), \\
\Gamma_{d_{i} d_{j}}^{A L R} & \simeq-i \frac{1}{\sqrt{2}} \epsilon_{i j}^{d}, \\
\Gamma_{u_{i} u_{j}}^{A L R} & \simeq i \frac{1}{\sqrt{2}} \epsilon_{i j}^{u}, \\
\Gamma_{u_{i} d_{j}}^{H^{+} L R} & \simeq V_{i j^{\prime}} \epsilon_{j^{\prime} j}^{d}, \\
\Gamma_{u_{i} d_{j}}^{H^{+} R L} & \simeq-\epsilon_{j^{\prime} i}^{u *} V_{j^{\prime} j}, \\
\Gamma_{\ell_{f} \ell_{i}}^{h L R} & \simeq \frac{\sin \alpha \tan \beta}{\sqrt{2}}\left(\frac{m_{\ell_{i}}}{v} \delta_{f i}-\epsilon_{f i}^{\ell}\right), \\
\Gamma_{\ell_{f} \ell_{i}}^{H L R} & \simeq-\frac{\cos \alpha \tan \beta}{\sqrt{2}}\left(\frac{m_{\ell_{i}}}{v} \delta_{f i}-\epsilon_{f i}^{\ell}\right), \\
\Gamma_{\ell_{f} \ell_{i}}^{A L R} & \simeq-i \frac{\tan \beta}{\sqrt{2}}\left(\frac{m_{\ell_{i}}}{v} \delta_{f i}-\epsilon_{f i}^{\ell}\right), \\
\Gamma_{v_{f} \ell_{i}}^{H^{+} L R} & \simeq \tan \beta\left(\frac{m_{\ell_{i}}}{v} \delta_{f i}-\epsilon_{f i}^{\ell}\right) .
\end{aligned}
$$

$V$ denotes the Cabibbo-Kobayashi-Maskawa mixing matrix, $v \simeq 174 \mathrm{GeV}$ the vacuum expectation value, and the $\epsilon$ matrices parametrize the deviation from the type-X structure ( $\epsilon^{f}$ and $\xi^{f}$ are related by fermion rotations). On phenomenological grounds, we take $\epsilon^{d}=0$ and both $\epsilon^{u}$ and $\epsilon^{\ell}$ of the form

$$
\epsilon^{u, \ell}=\left(\begin{array}{ccc}
0 & 0 & 0 \\
0 & 0 & 0 \\
0 & \times & \times
\end{array}\right),
$$

where $\times$ denotes a non-zero entry, allowing for lepton flavor violation in the $\mu-\tau$ sector.

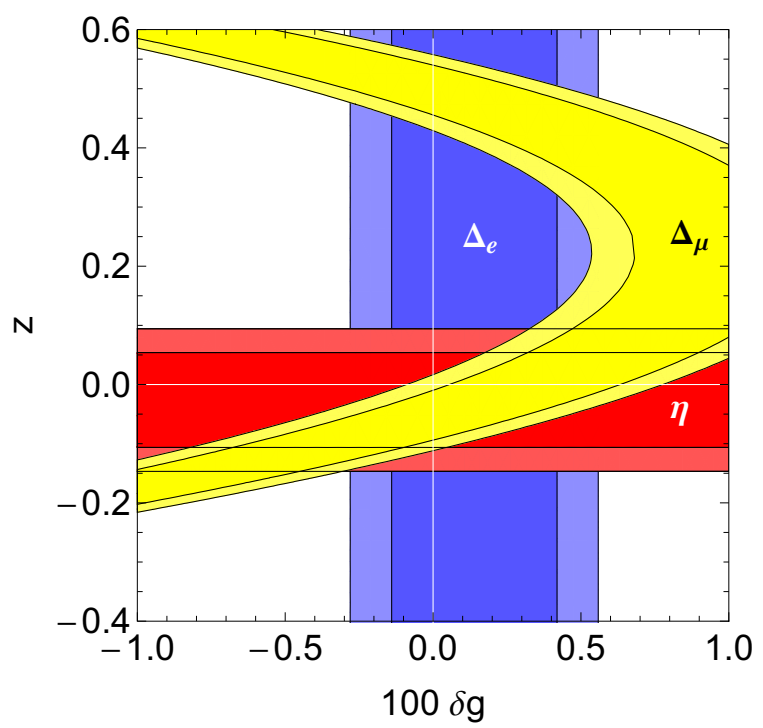

Figure 1. Allowed regions for $z$ and $\delta g$ from $\Delta_{\mu}$ (yellow), $\Delta_{e}$ (blue), and the Michel parameter $\eta$ (red); see text for definitions. Darker (lighter) regions are at the $2 \sigma(3 \sigma)$ level, using PDG values [1].

\section{Phenomenology}

In this section we address the constraints on our model and its potential to resolve the experimental anomalies outlined above.

\subsection{Tau decays $\tau \rightarrow \ell v v$}

Decays of the tau are modified at tree-level by the charged scalar $\mathrm{H}^{+}$(including a change in the Michel parameter $\eta)$ [24-26] and at loop-level through a modified $W \tau v$ coupling $g_{W \tau v} \rightarrow g_{W \tau v}(1+\delta g)[21,24,25]$ :

$$
\begin{aligned}
\Delta_{e} & \simeq 2 \delta g \\
\Delta_{\mu} & \simeq 2 \delta g+\frac{z^{2}}{4}-2 z \frac{m_{\mu}}{m_{\tau}}, \\
\eta & =-\frac{2 z}{4+z^{2}},
\end{aligned}
$$

with the 2HDM couplings [21]

$$
\begin{aligned}
z & \equiv \frac{v^{2}}{m_{H^{+}}^{2}} \Gamma_{v_{\tau} \tau}^{L R H^{+}} \Gamma_{v_{\mu} \mu}^{L R H^{+} \star}, \\
\delta g & =\frac{\tan ^{2} \beta}{32 \pi^{2}}\left|\frac{m_{\tau}}{v}-\epsilon_{33}^{\ell}\right|^{2}\left[F\left(\frac{m_{A}^{2}}{m_{H^{+}}^{2}}\right)+F\left(\frac{m_{H}^{2}}{m_{H^{+}}^{2}}\right)\right] .
\end{aligned}
$$

The experimentally allowed regions are shown in Fig. 1. In the SM we have $\delta g=z=0$, which is disfavored by $\tau \rightarrow$ $\mu v v$ at more than $2 \sigma$ (with PDG values). In the 2 HDM-X one has $\delta g \leq 0$ and $z>0$, which makes $\Delta_{\mu}$ even worse and puts pressure on the 2HDM-X and 2HDM-II (which has the same lepton couplings). In our modified 2HDM$\mathrm{X}$, we can however flip the sign of the tau coupling via $\epsilon_{33}^{\ell}>m_{\tau} / v$ and obtain $z<0$, alleviating the tension in $\tau \rightarrow \mu \nu v$ decays. 


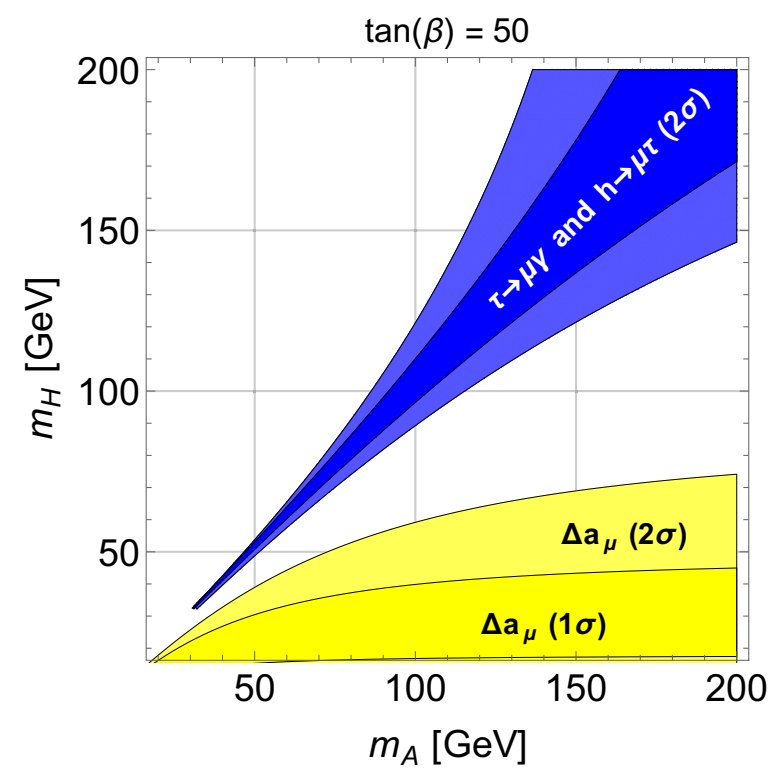

Figure 2. Allowed regions in the $m_{A}-m_{H}$ plane from $(g-2)_{\mu}$, $\tau \rightarrow \mu \gamma$ and $h \rightarrow \mu \tau$ for $\tan (\beta)=50$ and $\epsilon_{33}^{\ell}=2 m_{\tau} / v$. For $h \rightarrow \mu \tau$ blue corresponds to $\cos (\alpha-\beta)=0.1$ and light blue to $\cos (\alpha-\beta)=0.2$. The allowed region for $\Delta a_{\mu}$ is maximal in the sense that we have allowed for the three possibilities $\epsilon_{32}^{\ell} \neq 0$, $\epsilon_{32}^{\ell}=\epsilon_{23}^{\ell} \neq 0$ and $\epsilon_{32}^{\ell}=-\epsilon_{23}^{\ell} \neq 0$ as the latter ones can give $m_{\tau} / m_{\mu}$ enhanced one-loop contributions [1].

\subsection{Magnetic moment $a_{\mu}$ and $h \rightarrow \mu \tau$}

It has been shown that a light pseudoscalar in the 2HDM$\mathrm{X}$ with large $\tan \beta$ can resolve the $\Delta a_{\mu}$ anomaly via its contribution in a Barr-Zee diagram [36], see for example Refs. [19-21]. As seen above, we need to flip the sign of the tau coupling to the new scalars in order to alleviate the $\tau \rightarrow \mu v v$ discrepancy. Because of this, it is the non-SMlike CP-even scalar that can resolve the $\Delta a_{\mu}$ anomaly in our model (and not the pseudoscalar). The pseudoscalar then needs to be heavier than the scalar in order not to cancel the contribution to $\Delta a_{\mu}$ (see Fig 2).

The same Barr-Zee diagrams that give the desired $\Delta a_{\mu}$ also lead to $\tau \rightarrow \mu \gamma$ decays in case $\epsilon_{32}^{\ell} \neq 0$ (as required for $h \rightarrow \mu \tau$ ). If we want to explain $h \rightarrow \mu \tau$, the $\tau \rightarrow \mu \gamma$ rate needs to be tuned to small values using $m_{H} \simeq m_{A}$, which necessarily also suppresses the contribution to $\Delta a_{\mu}$ (see Fig 2). As a result, it is possible within our modified 2HDM-X to resolve the anomalies in $\tau \rightarrow \mu \nu v$ and $\Delta a_{\mu}$ or to resolve $\tau \rightarrow \mu \nu v$ and $h \rightarrow \mu \tau$, but not both at the same time, at least without introducing more parameters.

\subsection{Tauonic $B$ decays}

The relevant effective Hamiltonian for the semileptonic $B$ decays in our model is

$$
\mathcal{H}=C_{\mathrm{SM}}^{c b} \bar{c} \gamma_{\mu} P_{L} b \bar{\tau} \gamma_{\mu} P_{L} v_{\tau}+\sum_{X=L, R} C_{X}^{c b} \bar{c} P_{X} b \bar{\tau} P_{L} v_{\tau}
$$

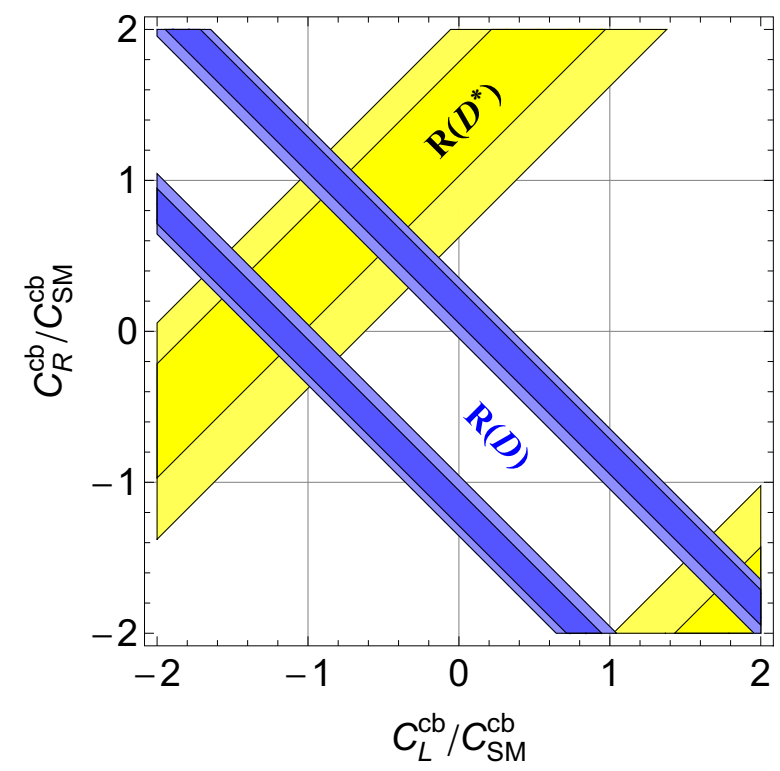

Figure 3. Allowed regions in the $C_{L}^{c b}-C_{R}^{c b}$ plane from $\mathcal{R}(D)$ (blue) and $\mathcal{R}\left(D^{*}\right)$ (yellow) for real values of $C_{L, R}^{c b}$. The lighter regions correspond to $2 \sigma$ experimental uncertainties while the darker regions are correspond to $1 \sigma$ [1].

with SM Wilson coefficient $C_{\mathrm{SM}}^{c b}=4 G_{F} V_{c b} / \sqrt{2}$ and the charged-scalar contribution

$$
C_{L}^{c b} \simeq \frac{\tan \beta}{m_{H^{+}}^{2}} \epsilon_{32}^{u *}\left(\frac{m_{\tau}}{v}-\epsilon_{33}^{\ell *}\right), \quad C_{R}^{c b} \simeq 0 .
$$

These Wilson coefficients affect the two ratios $\mathcal{R}\left(D^{(*)}\right)$ in the following way $[4,37,38]$,

$$
\begin{aligned}
& \frac{\mathcal{R}(D)}{\mathcal{R}(D)_{\mathrm{SM}}}=1+1.5 \mathfrak{R}\left[\frac{C_{R}^{c b}+C_{L}^{c b}}{C_{\mathrm{SM}}^{c b}}\right]+1.0\left|\frac{C_{R}^{c b}+C_{L}^{c b}}{C_{\mathrm{SM}}^{c b}}\right|^{2}, \\
& \frac{\mathcal{R}\left(D^{*}\right)}{\mathcal{R}\left(D^{*}\right)_{\mathrm{SM}}}=1+0.12 \mathfrak{R}\left[\frac{C_{R}^{c b}-C_{L}^{c b}}{C_{\mathrm{SM}}^{c b}}\right]+0.05\left|\frac{C_{R}^{c b}-C_{L}^{c b}}{C_{\mathrm{SM}}^{c b}}\right|^{2},
\end{aligned}
$$

leading to the allowed regions of Fig. 3. As can be seen, our new-physics model from Eq. (26) has the right structure to easily resolve the anomaly, e.g. with real $C_{L}^{c b} \simeq$ $-1.2\left|C_{\mathrm{SM}}^{c b}\right|^{1}$

\section{Conclusions}

We addressed the measured anomalies in $\mathcal{R}\left(D^{(*)}\right)(3.8 \sigma)$, $a_{\mu}(\sim 3 \sigma), \tau \rightarrow \mu \nu v(2.4 \sigma)$, and $h \rightarrow \mu \tau(2.6 \sigma)$ within a simple two-Higgs-doublet model. The Yukawa structure of our model is close to the lepton-specific $2 \mathrm{HDM}$

\footnotetext{
${ }^{1}$ Efficiency corrections to $\mathcal{R}(D)$ due to the BaBar detector [2] are important in the case of large contributions from $C_{R, L}^{c b}$, i.e. if one wants to explain $\mathcal{R}(D)$ with destructive interference with the SM contribution. As shown in Ref. [39], these corrections can be effectively taken into account by multiplying the quadratic term in $C_{R, L}^{c b}$ of Eq. (27) by an approximate factor of 1.5 (not included in Eq. (27)).
} 


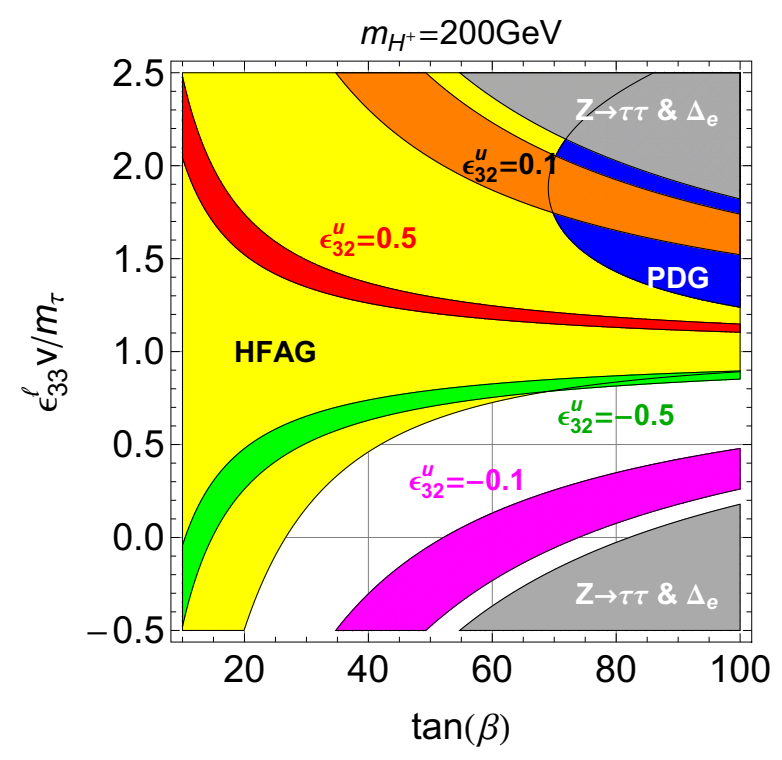

Figure 4. Allowed regions in the $\tan \beta-v / m_{\tau} \epsilon_{33}^{\ell}$ plane from $\mathcal{R}\left(D^{(*)}\right)$ and $\tau \rightarrow \mu \nu v$ at the $2 \sigma$ level. The yellow region is allowed by $\tau \rightarrow \mu v v$ using the HFAG result for $m_{H}=30 \mathrm{GeV}$ and $m_{A}=200 \mathrm{GeV}$, while the (darker) blue one is the allowed region using the PDG result. The red, orange, green, and magenta bands correspond to the allowed regions by $\mathcal{R}\left(D^{(*)}\right)$ for different values of $\epsilon_{32}^{u}$. The gray region is excluded by $Z \rightarrow \tau \tau$ and $\tau \rightarrow e v v$. For $m_{H} \simeq m_{A}$, the allowed regions from $\tau \rightarrow \mu \nu v$ would be slightly larger [1].

(type X), but with some additional Yukawa couplings involving third-generation fermions that give rise to the $b-c$ (necessary for $\mathcal{R}\left(D^{(*)}\right)$ ) and $\mu-\tau$ transitions (relevant for $h \rightarrow \mu \tau$ ) as well as corrections to $\tau \tau$ couplings (important for $\tau \rightarrow \mu v v)$.

The charged scalar $H^{+}$influences $\mathcal{R}\left(D^{(*)}\right)$ and $\tau \rightarrow$ $\mu \nu v$ and can resolve both deviations simultaneously (see Fig. 4), as long as the sign of the tau coupling is flipped, meaning $\epsilon_{33}^{\ell}>m_{\tau} / v$. Because of this, a light scalar (not pseudoscalar) is needed to resolve the $\Delta a_{\mu}$ anomaly, which then induces the top decay $t \rightarrow H c$ [1]. $h \rightarrow \mu \tau$ can only be explained if we give up on $\Delta a_{\mu}$, otherwise the rate $\tau \rightarrow \mu \gamma$ would be too large.

It remains to be seen which of these anomalies stand the test of time and which are simply statistical fluctuations. The fact that some of them can be explained rather naturally within a fairly minimal model gives however hope that we are on the verge of something interesting.

\section{Acknowledgements}

We thank the organizers of the FCCP2015 for an interesting workshop. A. Crivellin is supported by a Marie Curie Intra-European Fellowship of the European Community's 7th Framework Programme under contract number PIEFGA-2012-326948. The work of J. Heeck is funded in part by IISN and by Belgian Science Policy (IAP VII/37). P. Stoffer gratefully acknowledges financial support by the DFG (CRC 16, "Subnuclear Structure of Matter").

\section{References}

[1] A. Crivellin, J. Heeck, P. Stoffer (2015), 1507.07567

[2] J. Lees et al. (BABAR Collaboration), Phys.Rev.Lett. 109, 101802 (2012), 1205. 5442

[3] J.F. Kamenik, F. Mescia, Phys.Rev. D78, 014003 (2008), 0802. 3790

[4] S. Fajfer, J.F. Kamenik, I. Nisandzic, Phys.Rev. D85, 094025 (2012), 1203. 2654

[5] A. Crivellin, C. Greub, A. Kokulu, Phys.Rev. D86, 054014 (2012), 1206. 2634

[6] A. Celis, M. Jung, X.Q. Li, A. Pich, JHEP 1301, 054 (2013), 1210.8443

[7] A. Crivellin, A. Kokulu, C. Greub, Phys.Rev. D87, 094031 (2013), 1303. 5877

[8] G. Bennett et al. (Muon $(g-2)$ Collaboration), Phys.Rev. D73, 072003 (2006), hep-ex/0602035

[9] G. Colangelo, M. Hoferichter, M. Procura, P. Stoffer, JHEP 1409, 091 (2014), 1402 . 7081

[10] G. Colangelo, M. Hoferichter, B. Kubis, M. Procura, P. Stoffer, Phys. Lett. B738, 6 (2014), 1408 . 2517

[11] V. Pauk, M. Vanderhaeghen, Phys. Rev. D90, 113012 (2014), 1409.0819

[12] G. Colangelo, M. Hoferichter, M. Procura, P. Stoffer, JHEP 09, 074 (2015), 1506 . 01386

[13] M. Hayakawa, T. Blum, T. Izubuchi, N. Yamada, PoS LAT2005, 353 (2006), hep-lat/0509016

[14] T. Blum, M. Hayakawa, T. Izubuchi, PoS LATTICE2012, 022 (2012), 1301. 2607

[15] T. Blum, S. Chowdhury, M. Hayakawa, T. Izubuchi, Phys.Rev.Lett. 114, 012001 (2015), 1407.2923

[16] J. Green, O. Gryniuk, G. von Hippel, H.B. Meyer, V. Pascalutsa (2015), 1507.01577

[17] E.O. Iltan, H. Sundu, Acta Phys. Slov. 53, 17 (2003), hep-ph/0103105

[18] Y. Omura, E. Senaha, K. Tobe, JHEP 1505, 028 (2015), 1502.07824

[19] A. Broggio, E.J. Chun, M. Passera, K.M. Patel, S.K. Vempati, JHEP 1411, 058 (2014), 1409. 3199

[20] L. Wang, X.F. Han, JHEP 1505, 039 (2015), 1412.4874

[21] T. Abe, R. Sato, K. Yagyu, JHEP 07, 064 (2015), 1504.07059

[22] E.J. Chun, Z. Kang, M. Takeuchi, Y.L.S. Tsai (2015), 1507.08067

[23] K. Olive et al. (Particle Data Group), Chin.Phys. C38, 090001 (2014)

[24] M. Krawczyk, D. Temes, Eur.Phys.J. C44, 435 (2005), hep-ph/0410248

[25] W. Hollik, T. Sack, Phys.Lett. B284, 427 (1992)

[26] M. Aoki, S. Kanemura, K. Tsumura, K. Yagyu, Phys.Rev. D80, 015017 (2009), 0902 . 4665

[27] V. Khachatryan et al. (CMS), Phys. Lett. B749, 337 (2015), 1502.07400

[28] G. Aad et al. (ATLAS) (2015), 1508.03372 
[29] R. Harnik, J. Kopp, J. Zupan, JHEP 03, 026 (2013), 1209. 1397

[30] M.D. Campos, A.E.C. Hernández, H. Päs, E. Schumacher, Phys.Rev. D91, 116011 (2015), 1408. 1652

[31] D. Aristizabal Sierra, A. Vicente, Phys.Rev. D90, 115004 (2014), 1409. 7690

[32] J. Heeck, M. Holthausen, W. Rodejohann, Y. Shimizu, Nucl.Phys. B896, 281 (2015), 1412.3671

[33] A. Crivellin, G. D'Ambrosio, J. Heeck, Phys.Rev.Lett. 114, 151801 (2015), 1501.00993

[34] I. Dorsner, S. Fajfer, A. Greljo, J.F. Kamenik, N. Kosnik et al., JHEP 1506, 108 (2015),

\subsection{4}

[35] A. Crivellin, G. D’Ambrosio, J. Heeck, Phys.Rev. D91, 075006 (2015), 1503.03477

[36] S.M. Barr, A. Zee, Phys. Rev. Lett. 65, 21 (1990), [Erratum: Phys. Rev. Lett.65,2920(1990)]

[37] A. Akeroyd, S. Recksiegel, J.Phys.G G29, 2311 (2003), hep-ph/0306037

[38] Y. Sakaki, H. Tanaka, Phys.Rev. D87, 054002 (2013), 1205.4908

[39] S. Fajfer, J.F. Kamenik, I. Nisandzic, J. Zupan, Phys.Rev.Lett. 109, 161801 (2012), 1206. 1872 